\title{
Misdiagnosis of pulmonary artery aneurysm with eroding thrombus into the airways. A fatal case of suffocation
}

\author{
Ida Marie Svendsen ${ }^{1 *}$, Marina Rasmussen¹, Maria Høy Henningsen¹, Jytte Banner ${ }^{1}$
}

'Department of Forensic Pathology, Institute of Forensic Medicine, University of Copenhagen, Frederik V's Vej 11, 2100, Copenhagen East, Denmark

*Corresponding author ida.marie@dadlnet.dk

\begin{abstract}
:
We present a fatal case of hemoptysis following a thrombus-eroding pulmonary artery aneurysm into the left upper bronchus of a 79-year-old male with a history of multiple hospital contacts and examinations due to cough, hemoptysis, and reflux symptoms. A postmortem computed tomography (CT) scan revealed a hyperdense, condensed area in the left lung in relation to the lung hilus. At autopsy, the forensic specialist discovered a large, organized thrombus in a pulmonary artery aneurysm. The thrombus was adherent to the pulmonary artery aneurysm wall with an underlying defect directly communicating to the left upper bronchus. The cause of death was asphyxia due to blood in the airways (i.e., suffocation).

The combination of pulmonary artery hypertension, previous pulmonary embolism, and hemoptysis should lead to a particularly thorough inspection of the lungs with a focus on the pulmonary circulation. This case report emphasizes the importance of early detection of patients at risk of pulmonary artery rupture and attentiveness when performing biopsies during bronchoscopy to prevent communication between the artery and the airway. The risk of rupturing an aneurysm should be taken into account when performing biopsies on excrescence intruding into the bronchus in patients with medical histories of pulmonary hypertension, cough, and sporadic hemoptysis.
\end{abstract}

Keywords:

Hemoptysis, pulmonary artery aneurysm, aneurysm rupture, autopsy, case report

\section{INTRODUCTION}

Hemoptysis is a potentially fatal event that most often originates from the bronchial arterial system and is most often caused by bronchiectasis, chronic bronchitis, lung cancer or tuberculosis. In rare cases, hemoptysis originates from the pulmonic artery system, wherein a rupture of the pulmonary artery results in bleeding into the airways.

Rupture of a pulmonary artery is a rare but often fatal event. Such a rupture may occur due to dissection of the artery, an aneurysm, or a pseudoaneurysm. Due to its infrequency, the pulmonic artery system is seldom considered the origin of pathology in a patient with hemoptysia [1]. The pulmonary artery most often ruptures into the pericardium, resulting in hemopericardium; however, if the ruptured aneurysm is situated above the pericardial attachment, it will result in a hemorrhage into the surrounding tissue [2]-[4].

Pulmonary hypertension is associated with several complications, including dissection of the pulmonary artery, pulmonary artery aneurysm, right ventricular hypertrophy, and thrombus formation in the pulmonary circulation [2]. The diagnosis of a pulmonary aneurysm is of great importance in preventing a fatal rupture. However, the identification of patients with pulmonary artery aneurysms can be difficult because such aneurysms can present with unspecific symptoms, such as dyspnea, dysphonia, cough, cyanosis, compression symptoms, and retrosternal chest pain [5]-[7]. In cases of pulmonary artery rupture, the symptoms may depend on the location of the rupture: hemopericardium, hemorrhage in the lung parenchyma, or pronounced hemoptysis [8]-[10]. The majority of pulmonary artery dissections is caused by pulmonal hypertension, but a minor part occurs in aneurysm associated with atherosclerosis, congenital lesions or infections [6].

This case report illustrates the difficulties present when a pulmonary artery aneurysm is the infrequent etiology of hemoptysis and when the standardized medical examination of suspected lung cancer involves biopsy sampling during bronchoscopy.

\section{CASE REPORT}

A 79-year-old male was found dead in his bedroom in June 2018. He was lying in a pool of blood on the floor, and bloodstains were noticed in the bathroom sink. He had a medical history of systemic and pulmonary hypertension and pulmonary embolism. He had received medical treatment for his systemic hypertension, and an antemortem computed tomography (CT) scan of the thorax revealed infiltrates in the lungs and pleura as well as enlarged lymph nodes.

According to the deceased's medical record, he had undergone seven bronchoscopies over a five-year period from 2012 to 2017. A macroscopic normal bronchoscopy in April 2017 with endobronchial ultrasound guided biopsies, which showed no malignity or infection, was followed by three bronchoscopies with the identification of a bulging suspected cancerous 


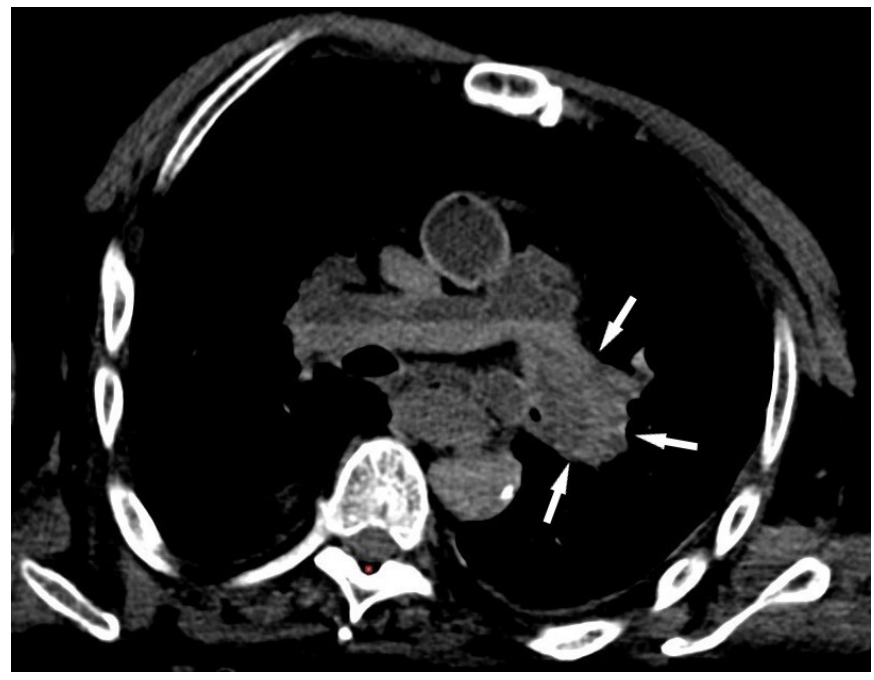

Figure 1: Transverse plane of the $\mathrm{CT}$, showing a diffuse condensed area in relation to the left pulmonary artery and the left bronchus possibly connecting two structures.

tumor in the left main bronchus. Two biopsies from this location showed granulation tissue but no signs of malignancy. The last hospital contact registered in the medical records was in January 2018, where a control CT scan showed an unchanged infiltrate in the right upper lobe of the lung and a few unchanged bilateral solid, subpleural, and peripheral nodules in the upper lobes of the lungs. The medical records documented treatment with blood-thinning medication due to multiple pulmonary embolisms in 2012. Deep venous thrombosis, pulmonary embolism and thrombosis was shortly described in the medical records in 2017, but with no further specifics. The last reported episode of hemoptysis occurred in November 2017. Furthermore, the deceased suffered from reflux due to hiatal herniation and was treated with proton pump inhibitors. No further medical records were available; thus, there were no available specific records of the bronchoscopies or radiological descriptions of the CT scans.

The external examination prior to autopsy revealed blood contamination on the face, trunk, and extremities. The oral cavity contained a dark, red liquid. There were no signs of violence.

\section{Postmortem computed tomography (PMCT) scan}

A postmortem CT (PMCT) scan prior to autopsy revealed esophageal herniation into the pulmonary cavity and dilatation of the duodenum. In relation to the left pulmonary artery and the left bronchus, a diffuse, condensed area was seen, possibly connecting two structures (Fig. 1). The lumen of the normally air-filled bronchus had a hyperdense content.

\section{Autopsy}

The autopsy revealed an organized thrombus adherent to the posterior wall of the left pulmonary artery, in a pulmonary artery aneurism (Fig. 2a). The dimensions of the thrombus were $3.5 \times 2.0$ and it did not obstruct the entire lumen of the artery but filled up the pulmonary aneurysm. In relation to the thrombus, the inferior wall of the artery had a $0.7-\mathrm{cm}$-long defect with direct
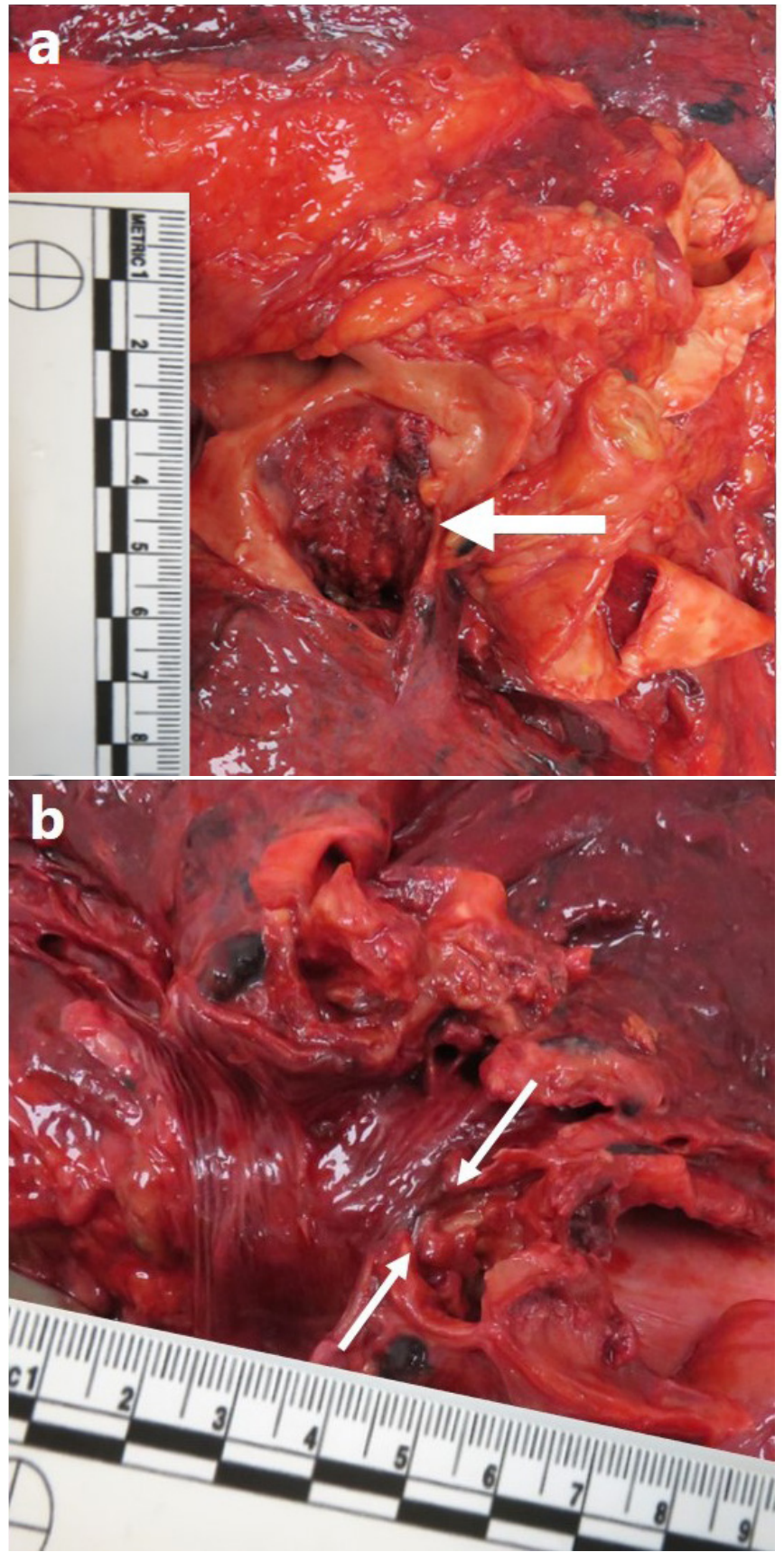

Figure 2: a Anterior view of the lungs, showing an organized thrombus attached to the posterior wall of the left pulmonic artery. The thrombus did not obstruct the entire lumen of the artery. b Posterior view of the upper left bronchus, showing a defect in the superior wall which is in direct communication with the inferior wall of the left pulmonic artery.

communication to the superior wall of the upper left bronchus (Fig. 2b). The defect in the bronchial wall showed rounded borders and signs of acute tissue reaction with redness, bleeding, and swelling. The airways presented with acute bronchial dilatation and contained blood. The pulmonary arteries had fatty streaks but no fibrous plaques, ulcers or calcification macroscopically. Part of the ventricle was situated in the right lung cavity due to the hiatal 


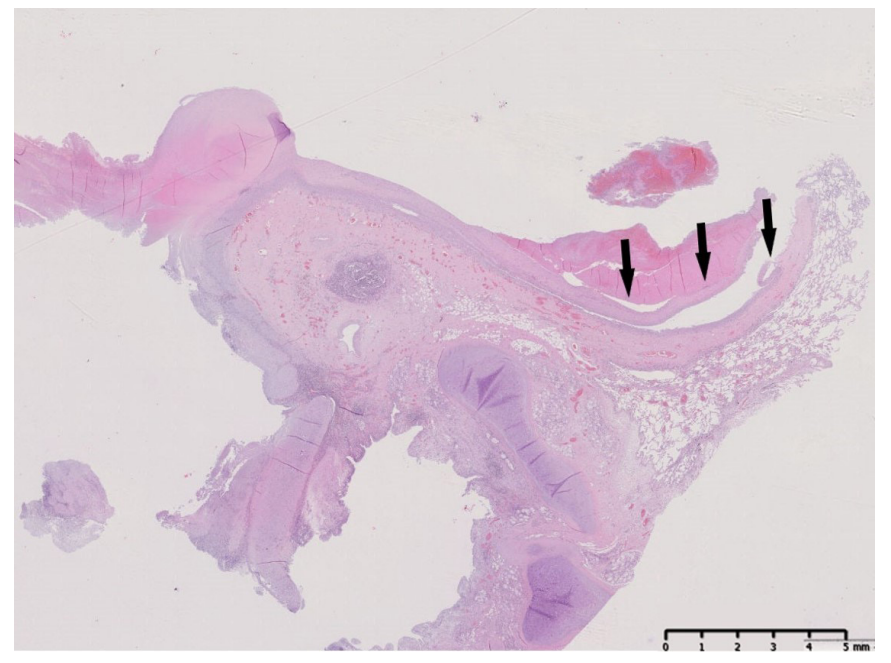

Figure 3: Histological image of the left pulmonic artery, showing thinning of the wall and in one area splitting of the tunica media with bleeding and inflammation.

herniation. The ventricle and the duodenum contained digested blood, and the heart was enlarged with a hypertrophic right ventricle.

The cause of death was suffocation due to blood in the airways.

\section{Histological analysis}

The histological analysis of the defect involving the left pulmonary artery and the adjacent bronchus showed pronounced areas with thinning of the artery wall and, in one area, splitting of the tunica media with bleeding and unspecific inflammation (Fig. 3). The artery wall was necrotic, with bleeding and granulation tissue at the borders. There was an impression of a bulge and, correspondingly, a fresh fibrin thrombus and surrounding accumulation of cholesterol crystals and fibrinous tissue, which could have comprised the edge of an atheromatous plaque. In one area, the structure of the arterial wall was completely abolished; correspondingly, a defect with bleeding and necrosis extended into the bronchial tissue between the circular cartilages. There were no signs of media necrosis, vasculitis or other degenerative changes.

The adjacent bronchial mucous membrane structure was eliminated, and necrosis was seen. Embedded in the necrosis was necrotic cartilaginous tissue and granulation tissue together with severe, diffuse unspecific inflammation, which spread out to the adjacent bronchial mucous membrane and bronchial tissue. There were no signs of specific inflammation, such as signs of tuberculosis.

The histological analysis was compatible with dissection of the pulmonary artery in relation to a smaller aneurysm with a possible atherosclerotic plaque and the development of a mural thrombus. Corresponding to this area was a penetration of the adjacent bronchial tissue with necrosis and bleeding, which supported the cause of death.

\section{DISCUSSION}

The autopsy revealed fatty streaks and no obvious atherosclerotic plaques in the pulmonary arteries. The histological analysis revealed a possible edge of an atheromatous plaque without calcification. It is therefore uncertain, whether the formation and rupture of the aneurism was a result of long-term stress to the artery wall, coursed by pulmonary hypertension alone, or a consequence of a possible underlying atherosclerotic plaques in the artery wall.

The small aneurysm might have eroded into the bronchus for some time before the rupture of the artery, as indicated by the rounded borders seen at autopsy. It is not known for how long the aneurysm intruded into the bronchus, but this could have been noticed in one of the multiple bronchoscopies. Taking the medical records into account, the suspected cancerous tumor found in the bronchoscopy in 2017 might have been a small aneurysm intruding into the left main bronchus. However, without the procedure description of the bronchoscopy, this is only a hypothesis. The biopsies might have contributed to thinning of the artery wall of the aneurysm, thereby making it weaker. A nearly identical case with an acute fatal outcome has been documented in the literature. In this case, a bronchoscopy was performed on a patient with lung symptoms. During the biopsy of a presumed polyp, the clinicians ruptured a pulmonary artery aneurysm, leading to massive bleeding and death [11]. In the present case, it is possible that had the aneurysm been thinner and/or had the biopsies been larger, a rupture of the aneurysm might have occurred during bronchoscopy.

Massive hemoptysis is a red flag for pulmonary artery rupture, often with a fatal outcome [6]. In the present case, the deceased had pulmonary hypertension, cough, and occasional hemoptysis and had undergone seven bronchoscopies, none of which resulted in a diagnosis. These symptoms, together with the negative bronchoscopies, could have pointed in the direction of a pulmonary circulation etiology and more relevant diagnostic tests, such as transthoracal or transesophagal echocardiography, angiography, a contrast-enhanced CT scan, or a magnetic resonance imaging (MRI) scan [12].

Considering the size of the defect, it might have been difficult to visualize on a CT scan. The PMCT showed a condensed area between the left pulmonary artery and the left bronchus, which possibly could have been discovered on a CT scan and helped the diagnosis. In the literature, pulmonary artery aneurysms have been discovered using contrastenhanced $\mathrm{CT}$, but these aneurysms seem to have been larger than that in the present case [12]-[15]. According to the available medical records, the deceased had undergone several CT scans. However, there were no radiological descriptions of the types or pathological findings of these scans. It is possible that the characteristics of one or more of these $\mathrm{CT}$ scans could have indicated that the deceased had an aneurysm intruding into the bronchus. Therefore, this case emphasizes the importance of retrieving medical records through the police authority in cases in which the cause of death relates to a specific medical procedure.

We hope that this case report will contribute to an increased awareness of pulmonary artery aneurysms and help to prevent medical misdiagnosis and consequent mistreatment in the future.

\section{DECLARATIONS}

Conflicts of interest/Competing interests: Not applicable

\section{CONSENT FOR PUBLICATION}

The relatives of the deceased approves the publication of this case report. 


\section{REFERENCES}

[1] A. Khalil, B. Fedida, A. Parrot, S. Haddad, M. Fartoukh, and M. F. Carette, "Severe hemoptysis: From diagnosis to embolization," Diagn. Interv. Imaging, vol. 96, no. 7-8, pp. 775-788, 2015, doi: 10.1016/j.diii.2015.06.007.

[2] S. M. Mak, N. Strickland, and D. Gopalan, "Complications of pulmonary hypertension: a pictorial review.," Br. J. Radiol., vol. 90, no. 1070, p. 20160745, Feb. 2017, doi: 10.1259/bjr.20160745.

[3] J. B. McNaught and W. Dock, "Spontaneous Rupture of the Pulmonary Artery.," Am. J. Pathol., vol. 11, no. 6, pp. 989-1000.3, Nov. 1935, Accessed: Sep. 19, 2018. [Online]. Available: http://www.ncbi.nlm.nih.gov/pubmed/19970246.

[4] P. a Thomason and K. R. Krach, "Spontaneous rupture of the pulmonary artery.," Ann. Emerg. Med., vol. 17, no. 1, pp. 63-5, 1988, [Online]. Available: http://www.ncbi.nlm. nih.gov/pubmed/3337418.

[5] P. Theodoropoulos, B. A. Ziganshin, M. Tranquilli, and J. A. Elefteriades, "Pulmonary Artery Aneurysms: Four case Reports and literature review.," Int. J. Angiol., vol. 22, no. 3, pp. 143-8, Sep. 2013, doi: 10.1055/s-0033-1347907.

[6] C. Zhang, X. Huang, S. Li, H. Yao, and B. Zhang, "Pulmonary Artery Dissection: A Fatal Complication of Pulmonary Hypertension," Case Rep. Med., vol. 2016, pp. 1-5, Nov. 2016, doi: 10.1155/2016/4739803.

[7] B. Degano, G. Prevot, L. Têtu, O. Sitbon, G. Simonneau, and M. Humbert, "Fatal dissection of the pulmonary artery in pulmonary arterial hypertension.," Eur Respir Rev, vol. 18, no. 113, pp. 181-5, Sep. 2009, doi: 10.1183/09059180.00002909.

[8] K. Ohela and H. Teir, "Rupture of the pulmonary artery; report of three cases., Ann. Med. Intern. Fenn., vol. 43, no. 1, pp. 39-44, 1954, Accessed: Sep. 19, 2018. [Online]. Available: http://www.ncbi.nlm.nih.gov/pubmed/13158983.
[9] D. Durak, B. Eren, N. Turkmen, and R. Fedakar, "Pulmonary artery aneurysm rupture., Bratisl. Lek. Listy, vol. 109, no. 12, pp. 582-3, 2008.

[10] E. C. Seyhan, M. Z. Gunluoglu, and C. Erol, “Pulmonary Artery Aneurysm Ruptured

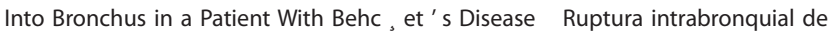
un aneurisma de arteria pulmonar en un paciente con enfermedad de behc," Arch. Bronconeumol. (English Ed., vol. 53, no. 1, p. 2129, Jan. 2017, doi: 10.1016/j. arbr.2016.03.014

[11] J. H. Park, Y. Lee, Y. Oh, and S. Oh, "Death after Bronchoscopic Biopsy of a Pulmonary Artery Aneurysm Mimicking Bronchial Polyp," Korean J. Leg. Med., pp. 73-77, 2017.

[12] M. Kreibich, M. Siepe, J. Kroll, R. Höhn, J. Grohmann, and F. Beyersdorf, “Aneurysms of the pulmonary artery," Circulation, vol. 131, no. 3, pp. 310-316, Jan. 2015, doi: 10.1161/CIRCULATIONAHA.114.012907.

[13] H. H. Shih, P. L. Kang, C. Y. Lin, and Y. H. Lin, "Main pulmonary artery aneurysm," J. Chinese Med. Assoc., vol. 70, no. 10, pp. 453-455, Oct. 2007, doi: 10.1016/S17264901(08)70038-8.

[14] D. Puri, H. P. Kaur, R. Brar, K. P. Singh, M. Sahoo, and T. S. Mahant, "Ruptured pulmonary artery aneurysm: A surgical emergency," Asian Cardiovasc. Thorac. Ann., vol. 19, no. 6, pp. 436-439, Dec. 2011, doi: 10.1177/0218492311421443.

[15] M. Seguchi et al., "Idiopathic pulmonary artery aneurysm," Circulation, vol. 124, no. 14. Oct. 04, 2011, doi: 10.1161/CIRCULATIONAHA.111.029033. 